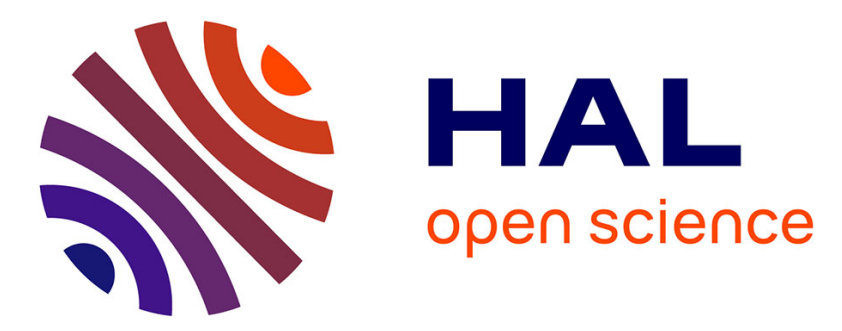

\title{
Un outil en os à usages multiples dans un contexte moustérien
}

\author{
Maurice Hardy, Geneviève Pothier Bouchard, Luc Doyon
}

\section{To cite this version:}

Maurice Hardy, Geneviève Pothier Bouchard, Luc Doyon. Un outil en os à usages multiples dans un contexte moustérien. Bulletin de la Société préhistorique française, 2014, 111 (4), pp.741-744. hal-02864085

\section{HAL Id: hal-02864085 \\ https://hal.science/hal-02864085}

Submitted on 29 Sep 2021

HAL is a multi-disciplinary open access archive for the deposit and dissemination of scientific research documents, whether they are published or not. The documents may come from teaching and research institutions in France or abroad, or from public or private research centers.
L'archive ouverte pluridisciplinaire HAL, est destinée au dépôt et à la diffusion de documents scientifiques de niveau recherche, publiés ou non, émanant des établissements d'enseignement et de recherche français ou étrangers, des laboratoires publics ou privés. 


\title{
ACTUALITÉS SCIENTIFIQUES
}

\author{
DÉCOUVERTES RÉCENTES
}

\section{Un outil en os à usages multiples dans un contexte moustérien}

\author{
Maurice Hardy, Geneviève Pothier Bouchard et Luc Doyon
}

\begin{abstract}
T A PRODUCtion teChnOlogiQue sur supports osseux

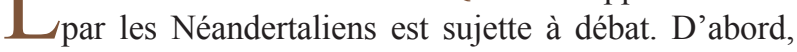
pendant la majeure partie du $\mathrm{Xx}^{\mathrm{e}}$ siècle, les préhistoriens furent réticents à reconnaître à cette espèce la capacité d'intégrer ce type de matière première dans son répertoire technologique. Par la suite, des doutes furent émis quant à la capacité de l'homme de Néandertal à maîtriser un ensemble de techniques propres au travail de l'os. Au cours des deux dernières décennies, cette position concernant l'outillage osseux du Paléolithique moyen européen se voit sensiblement modifiée, tant par la révision critique des données stratigraphiques que par la publication d'indices toujours plus nombreux corroborant l'utilisation des matières dures d'origine animale dans le cadre d'activités techniques par les Néandertaliens. La présente communication participe à ce mouvement de remise en question par la présentation de la découverte d'un témoin supplémentaire du travail de l'os par l'homme de Néandertal. Mise au jour lors de la mission de fouille 2014 de la grotte du Bison à Arcy-sur-Cure (Yonne, France), la pièce correspond à un outil à usages multiples sur fragment proximal de diaphyse d'un fémur de renne (Rangifer tarandus).
\end{abstract}

\section{Le site et historique des fouilles}

Découverte en 1958 grâce à la présence d'un passage comblé dans la paroi occidentale de la grotte du Renne, la grotte du Bison révéla plusieurs sols d'occupations (sondages P. Poulain) s'échelonnant du Moustérien (E-F : Moustérien récent; G-H : Moustérien à denticulés; I-J : Moustérien typique ancien) au Châtelperronien (D; Leroi-Gourhan, 1961). Elle fait partie des onze grottes occupées parmi les treize s'ouvrant au sud dans la barrière corallienne qui borde le Bassin parisien. À cet endroit, la Cure franchit ses derniers méandres après être sortie du Morvan.

$\mathrm{Au}$ cours des premières campagnes dirigées par A. Leroi-Gourhan (1959-1963), les niveaux moustériens de la grotte du Bison n'ont fait l'objet que de travaux limités en surface (Girard, 1982). Depuis 1995, les fouilles ont repris, sous la direction de F. David (1995-2010) puis de M. Hardy (depuis 2011), afin de compléter les informations déjà recueillies (Lhomme et al., 2005; David et al., 2006 et 2009; Enloe, 2013; Tillier et al., 2013a).
La grotte s'ouvre au sommet d'une petite falaise, à six mètres environ au-dessus de la rive. Elle comporte trois parties : 1) au sud, l'Avant, d'une trentaine de mètres carrés, qui livre des traces évidentes d'activités humaines (outillage lithique abondant, galets, ocre); 2) au centre, le Parvis, un vaste espace d'environ $50 \mathrm{~m}^{2}$ dont le plafond s'est effondré; 3 ) au nord, la grotte se prolonge par une Galerie de 4,5 m de long, 1,8 $\mathrm{m}$ de large et $0,6 \mathrm{~m}$ de haut (au plus haut), où se sont déroulées des activités liées à plusieurs zones de combustion mises au jour.

Depuis 2012, les vestiges archéologiques de la couche $\mathrm{J}$ sont mis au jour. L'assemblage lithique comprend plus de 150 outils (éclats à retouches marginales, racloirs simples, doubles ou convergents, denticulés, encoches, pièces esquillées et nucléus). La production met en œuvre un débitage Levallois d'organisation centripète ou unipolaire-bipolaire parallèle, tant sur silex que sur chaille. Les nombreux éclats de débitage et de réaffûtage mis au jour attestent de la mise en forme et du ravivage des outils sur le site d'occupation (Hardy et al., 2013).

La couche $\mathrm{J}$ a également livré un nombre important de vestiges fauniques dont plus de 3250 éléments identifiables. L'ensemble est composé de cheval, de renne et de bison à plus de $80 \%$. En sus de ces taxons, on trouve aussi l'hyène des cavernes, l'ours des cavernes, le loup et le renard ainsi que d'autres espèces typiques de la faune quaternaire associées à un environnement de steppe fraîche ou de forêt claire. Les éléments squelettiques présentent un état de conservation variable suivant leur position dans la grotte : altérés à l'Avant, assez souvent écrasés sur le Parvis par la chute de blocs de calcaire tombés de la voûte, ils sont en bon état dans la Galerie.

La présence humaine est attestée dans la couche $\mathrm{J}$ par neuf dents néandertaliennes isolées : quatre ayant fait l'objet d'une publication (Tillier et al., 2013b), deux trouvées en 2013 (Hardy et al., 2013) et trois trouvées en 2014 (Hardy et al., à paraître). On trouve également des galets sélectionnés selon leur taille de même qu'une zone, à l'Avant, dans un renfoncement situé à l'est, ayant livré une multitude de nodules d'ocre $(30 \mathrm{~kg}$; masse moyenne : $65 \mathrm{~g})$. Les vestiges archéologiques sont surtout distribués le long de la paroi est; les activités humaines y furent probablement plus intenses, la paroi ouest servant plutôt de zone de rejet des restes fauniques. 


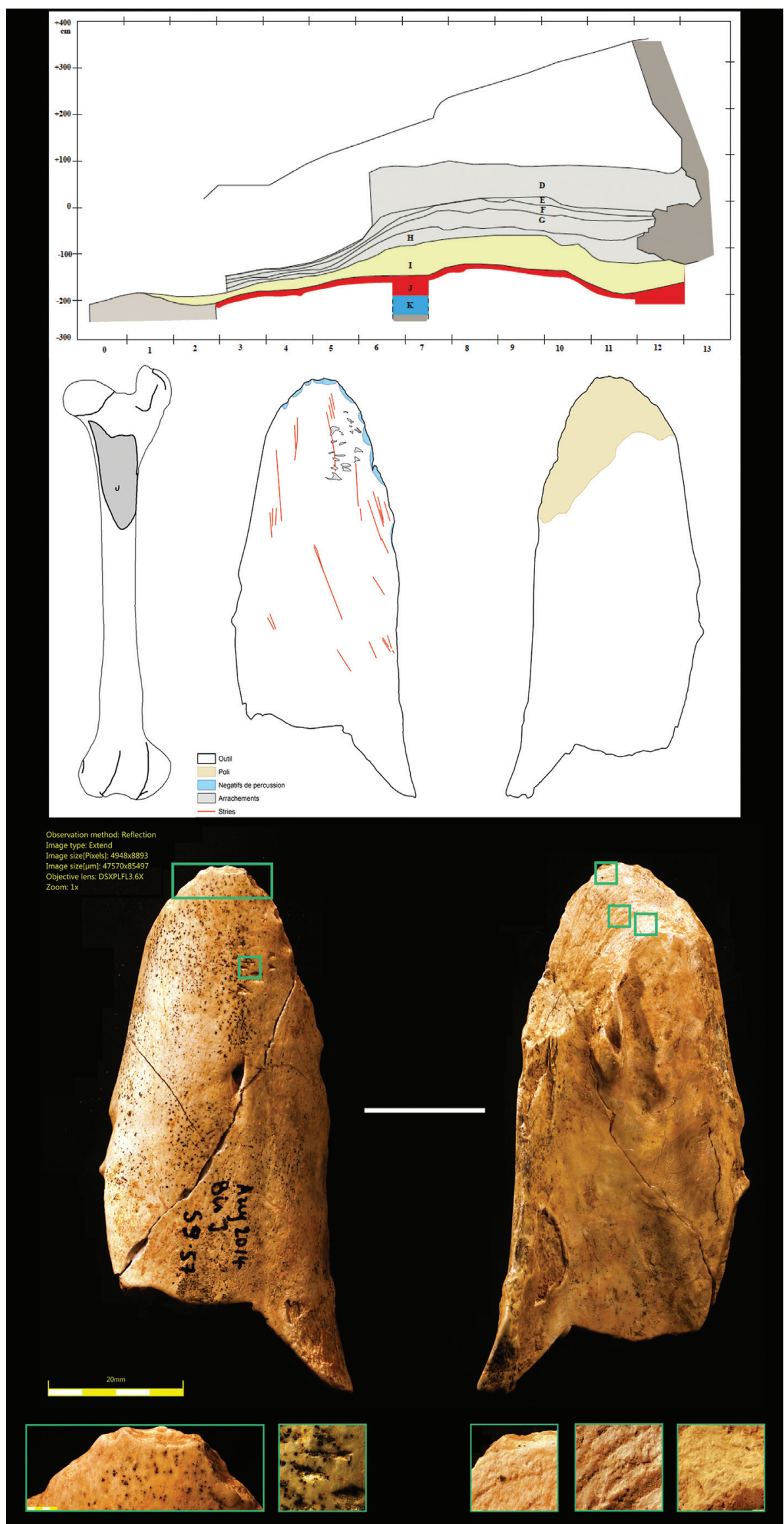

Fig. 1 - Haut : profil stratigraphique de la grotte du Bison (A. Roblin-Jouve in Hardy et al., 2013); centre : origine anatomique et schéma technique de l'outil en os (S9.57); bas : macro- et microscopie de l'outil. 
La couche J n'a livré aucune trace d'habitation moustérienne à l'exception des trois foyers avérés dans la Galerie. La mission 2014 a d'ailleurs permis la découverte de deux nouvelles structures de combustion, l'une au nord-est, l'autre au sud-est de cette caverne.

La nature des vestiges et leur distribution spatiale nous interpellent : depuis quelques années, nous pressentons que le site a été employé en tant qu'atelier de boucherie et de peausserie. Dans ce contexte, la Galerie a pu servir de fumoir. Les analyses tracéologiques à venir, tant sur l'ocre que sur le mobilier lithique, permettront sans doute de tester cette hypothèse.

\section{L'outil}

Trouvé contre la paroi est sur le Parvis, le fragment osseux provient de la face crâniale du premier tiers proximal de la diaphyse d'un fémur gauche de renne adulte. La pièce, retrouvée brisée, présente un excellent état de conservation. Aucune altération liée à l'action des carnivores n'est apparente. Une légère coloration, sous forme de petites taches noires, traduit l'altération ferromanganique causée par la stagnation de l'eau en milieu karstique. On les retrouve principalement sur la surface corticale de l'os sans toutefois qu'elles masquent les traces anthropiques.

L'analyse des stigmates présents sur le fragment permet de retracer son utilisation dans quatre activités distinctes. Dans un premier temps, vingt-neuf traces de décarnisation sont visibles sur la surface supérieure de l'outil. Dans la majorité des cas, ces stigmates sont orientés longitudinalement par rapport à la diaphyse avec une déviation vers la vue médiale de l'élément squelettique; ils suggèrent la nature anthropique de l'exploitation des ressources carnées.

Dans un second temps, les pans de la diaphyse laissent supposer une fracturation de l'os frais. L'objectif principal de la fracture a pu être l'extraction de la moelle, l'activité produisant un support ayant une morphologie idéale pour sa transformation en outil.

Dans un troisième temps, vingt-deux traces d'arrachement subparallèles les unes par rapport aux autres et disposées transversalement à l'axe de l'outil sur la partie distale de sa face supérieure suggèrent l'emploi de la diaphyse en tant que retouchoir pour la taille de produits lithiques.

Finalement, l'objet porte dix négatifs d'enlèvement sur sa face supérieure ainsi qu'un poli d'utilisation sur sa face inférieure. Ces traces délimitent l'arête active de l'extrémité distale de l'outil. Il n'est pas possible d'écarter l'hypothèse que la modification morphologique du fil de l'outil soit due à son utilisation. Toutefois, la régularité des enlèvements le long de l'arête peut être le résultat d'une mise en forme intentionnelle du fil dont l'utilisation anthropique effaça les traces. Si tel est le cas, il serait logique de croire que les enlèvements visaient à accroître les propriétés de lacération de l'apex.

La lecture technologique permet d'avancer l'hypothèse qu'il s'agit d'un outil en os à usages multiples ayant servi comme retouchoir et racloir. L'approche expérimentale pourra tester cette hypothèse.

\section{Discussion}

La caractérisation technologique de la pièce confirme que les Néandertaliens connaissaient les propriétés minérales de l'os comme matière première exploitable pour la fabrication d'outils. La technique de fabrication observée pour la production du racloir suggère un transfert des gestes associés à la taille d'un support lithique vers un support sur matière dure animale. Considérant cette adaptation technique et la rareté de ce type d'outil associé à la culture moustérienne, il est essentiel de s'interroger sur la disponibilité des ressources de matière première lithique. La présence de cet outil dans un contexte où l'industrie lithique abonde et l'observation d'un important poli sur sa face inférieure laissent croire à un choix intentionnel du support par les Néandertaliens.

Le caractère unique de l'objet au sein du matériel archéologique de l'ensemble karstique d'Arcy-sur-Cure peut s'expliquer par l'état général de conservation des collections fauniques. Les facteurs taphonomiques restreignent souvent l'analyse tracéologique des restes osseux. À cet effet, soulignons l'alternance d'occupations entre l'hyène des cavernes et les Néandertaliens attestée à la grotte du Bison (Enloe, 2012). Ce phénomène engendre une forte altération de l'ensemble des restes fauniques par l'action de l'hyène, en plus de celles attribuables aux autres carnivores tels le loup et le renard. Finalement, à ceci s'ajoutent les altérations naturelles associées au contexte des sites en grotte dont la fragmentation des vestiges osseux, le concrétionnement et le ruissellement.

Il ne faut pas oublier qu'en plus des facteurs liés aux processus de formation des dépôts sédimentaires, l'orientation des études archéozoologiques peut être déterminante dans la reconnaissance d'une industrie osseuse moustérienne. En complétant l'analyse faunique par une approche technoéconomique, il devient possible d'interpréter en termes de comportements anthropiques des stigmates difficilement perceptibles ou même parfois confondus avec l'action des agents taphonomiques.

L'analyse et la discussion qui précèdent offrent deux perspectives de recherches jusqu'à maintenant peu exploitées. Il devient nécessaire de revoir les collections fauniques moustériennes déjà mises au jour à Arcy-surCure. L'élaboration d'un projet expérimental permettra une meilleure compréhension du schéma de débitage, de transformation et d'utilisation d'un tel outil.

Remerciements : Nous remercions M. François de la Varende d'avoir accordé l'autorisation de fouilles à la grotte du Bison et Francine David pour la transmission de ses connaissances ostéologiques. Nous soulignons également le concours des $\mathrm{D}^{\mathrm{r}} \mathrm{A}$. Burke, M. Drapeau, I. Ribot et Y. Chebli pour avoir facilité l'accès au macroscope DSX100 (Olympus) du laboratoire de paléoanthropologie et d'écomorphologie de l'université de Montréal. 


\section{RÉFÉRENCES BIBLIOGRAPHIQUES}

David F., Connet N., Girard M., Miskovsky J.-C., MourerChauviré C., Roblin-Jouve A. (2006) - Les niveaux du Paléolithique supérieur à la grotte du Bison (Arcy-sur-Cure, Yonne) : couches a à d, Revue archéologique de l'Est, 54, p. 5-50.

David F., D'Iatchenko V., Enloe J. G., Girard M., Hardy M., Lhomme V., Roblin-Jouve A., Tillier A., Tolmie C. (2009) - New Neandertal Remains from the Grotte du Bison at Arcy-sur-Cure, France, Journal of Human Evolution, 57, 6, p. 805-809.

Enloe J. G. (2012) - Middle Palaeolithic Cave Taphonomy: Discerning Humans from Hyenas at Arcy-sur-Cure, France, International Journal of Osteoarchaeology, 22, 5, p. 591-602.

Enloe J. G. (2013) - Neanderthal to Neanderthal Evolution: Preliminary Observations on Faunal Exploitation from Mousterian to Châtelperronian at Arcy-sur-Cure, in J. L. Clark et J. D. Speth (dir.), Zooarchaeology and Modern Human Origins, Dordrecht, Springer Netherlands (Vertebrate Paleobiology and Paleoanthropology), p. 163-172.

GIRARD C. (1982) - Les industries moustériennes de la grotte du Bison à Arcy-sur-Cure (Yonne), Gallia Préhistoire, 25, 1, p. 107-129.

Hardy M., David F., Doyon L., D'Iatchenko V. I., Girard M., Lhomme V., Pothier Bouchard G. (à paraître) - Arcy-sur-Cure (Yonne). Grotte du Bison, Rapport 2014, rapport de fouille, service régional de l'Archéologie de Bourgogne, Dijon.

Hardy M., David F., Doyon L., D'Iatchenko V. I., Girard M., Lhomme V., Pothier Bouchard G., RoblinJouve A. (2013) - Arcy-sur-Cure (Yonne). Grotte du
Bison, Rapport 2013, rapport de fouille, service régional de l'Archéologie de Bourgogne, Dijon, 96 p.

Leroi-Gourhan A. (1961) - Les fouilles d'Arcy-sur-Cure (Yonne), Gallia Préhistoire, 4, 1, p. 3-16.

Lhomme V., David F., Thiébaut C. (2005) - Les industries de la fin du Paléolithique moyen de la grotte du Bison à Arcy-sur-Cure (Yonne), in N. Molines, M.-H. Moncel et J.-L. Monnier (dir.), Données récentes sur les modalités de peuplement et sur le cadre chronostratigraphique, géologique et paléogéographique des industries du Paléolithique inférieur et moyen en Europe, Oxford, Archaeopress (BAR, International Series 1364), p. 479-498.

Tillier A.-M., Hardy M., David F., Girard M., D'Iatchenko V. (2013a) - À propos de deux molaires déciduales inférieures provenant des niveaux moustériens de la grotte du Bison (Arcy-sur-Cure, Yonne, France), Paleo, 24, p. 271-278.

Tillier A.-M., Sansilbano-Collilieux M., David F., Enloe J. G., Girard M., Hardy M., D'Iatchenko V., Roblin-Jouve A., Tolmie C. (2013b) - Les vestiges néanderthaliens provenant des niveaux moustériens $\mathrm{I}$ et $\mathrm{J}$ de la grotte du Bison à Arcy-sur-Cure (Yonne) : bilan actuel, Bulletins et mémoires de la Société d'anthropologie de Paris, 25, 1-2, p. 39-54.

Maurice HARDY maurice.hardy@mae.u-paris10.fr

Geneviève Pothier Bouchard genevieve.pothier.bouchard@umontreal.ca

Luc Doyon luc.doyon@umontreal.ca 